\title{
Análise do Desempenho de Heurísticas na Coleta de Informações de Telemetria In-Band*
}

\author{
Ariel G. de Castro ${ }^{1}$, Francisco G. Vogt ${ }^{1}$, Victor H. S. Lopes ${ }^{1}$, \\ Sérgio R. B. Silva ${ }^{1}$, Arthur F. Lorenzon ${ }^{1}$, Fábio D. Rossi ${ }^{2}$, Marcelo C. Luizelli ${ }^{1}$ \\ ${ }^{1}$ Universidade Federal do Pampa (UNIPAMPA) \\ ${ }^{2}$ Instituto Federal Farroupilha (IFFAR)
}

\begin{abstract}
In-band network telemetry has emerged as an alternative to traditional monitoring. It allows to obtain telemetry data with higher granularity from the data plane, which increases the level of visibility to the operator. However, little has yet been done to orchestrate how in-band network telemetry data is collected from the network. In this paper, we propose two online heuristics to orchestrate how telemetry data is collected. Results show that the proposed heuristics outperform existing solutions, being able to collect telemetry data up to $30 \%$ more frequently.
\end{abstract}

Resumo. A telemetria in-band surgiu recentemente como uma alternativa ao monitoramento tradicional e permite encapsular informações de monitoramento com uma granularidade mais fina a partir do plano de dados - permitindo aumentar o nível de visibilidade fornecido ao operador. Nesse sentido, pouco ainda foi feito para orquestrar a coleta de dados relacionados a telemetria in-band. Neste artigo, propõe-se duas heurísticas online para coletar informações de telemetria baseadas nas estratégias round-robin e least recently collected. Resultados mostram que as heurísticas projetadas conseguem coletar os dados de telemetria com $30 \%$ a mais de frequência e com maior uniformidade ao longo do tempo que as abordagens existentes.

\section{Introdução}

A telemetria in-band é um paradigma emergente de monitoramento de infraestruturas de rede que permite maior flexibilidade na coleta dos dados [Q. Wu and Zhang. 2016]. Permite-se, por meio da programação de dispositivos de encaminhamento, a inclusão de informações de monitoramento (por exemplo, tamanho da fila de um dispositivo, tempo de processamento de um pacote) nos próprios pacotes dos tráfegos de rede ativos. Essas informações fornecem maior cobertura sobre o estado da rede, auxiliando no gerenciamento da infraestrutura de rede e suas aplicações [Tammana et al. 2018, Jianzhe Liang 2018], [Gulenko et al. 2018].

Com a utilização de telemetria in-band, é possível aumentar o nível de visibilidade (isto é, cobertura dos dispositivos de rede) e ajustar a granularidade desejada sobre a coleta dos dados. Um maior nível de visibilidade da infraestrutura de rede permite que uma gama maior de problemas e anomalias como, por exemplo, detecção de heavy hitters e de ataques DDoS [Tammana et al. 2016, Liu et al. 2016] sejam identificados com maior acurácia em comparação a abordagens tradicionais (por exemplo, protocolo SNMP). A coleta dos dados de telemetria é feita de forma sistemática, auxiliando o operador, que passa a possuir maior controle sobre o estado da rede, de forma a atender às necessidades de diferentes aplicações com a granularidade desejada.

Abordagens recentes [Putina et al. 2018], [Gupta et al. 2018], [Liu et al. 2018], [Tammana et al. 2018], [Marques et al. 2019] têm focado em utilizar os dados de telemetria para identificar e prever comportamentos anômalos em infraestruturas de

*Suportado por CNPq e FAPERGS 
rede. Entretanto, pouco ainda foi feito para orquestrar como os dados devem ser coletados em tais infraestruturas. Orquestrar a coleta dos itens é particularmente desafiador por dois principais motivos. Primeiramente, dependendo de quais itens são coletados, a visibilidade da rede pode ser degradada em termos de consistência. Segundo, dependendo de como os itens são coletados, o desempenho da rede pode ser degradado. Em [Marques et al. 2019], os autores propõem uma abordagem para a orquestração da coleta de itens de telemetria baseada em duas heurísticas: (i) Concentra e (ii) Distribui. Apesar deste primeiro esforço na direção da orquestração eficiente da dados de telemetria, as soluções apresentadas são estáticas (computadas de maneira offline) ao longo do tempo.

Nesse artigo, propõe-se duas heurísticas online para realizar a orquestração de dados de telemetria - isto é, determinar quais fluxos de rede coletam quais dados de telemetria. A primeira heurística baseia-se no conceito Least Recently Collected (LRC), enquanto que a segunda em Round-Robin $(R R)$. As heurísticas propostas são comparadas com o trabalho de [Marques et al. 2019]. Na avaliação, consideram-se as métricas (i) quantidade de itens de telemetria coletados e cobertura, (ii) tempo médio para a coleta dos dados de telemetria e (iii) distribuição do número de coletas ao longo do tempo. A partir da análise dos resultados, observa-se que o algoritmo LRC consegue coletar dados de telemetria de maneira mais frequente (até $30 \%$ em relação as outras abordagens) e com maior uniformidade ao longo do tempo.

O restante do artigo está organizado como segue. Na Seção 2, descreve-se os trabalhos mais recentes relacionados à telemetria in-band. Na Seção 3, o problema da coleta de itens de telemetria é formalmente definido. Na Seção 4, apresenta-se as heurísticas propostas: $L R C$ e $R R$. Os resultados são discutidos na Seção 5. Por fim, conclui-se o trabalho com as considerações finais e perspectivas de trabalhos futuros na Seção 6.

\section{Trabalhos Relacionados}

Para manter a visibilidade e o freshness da infraestrutura de rede, é necessário coletar itens de telemetria de forma a mitigar seu impacto sobre o desempenho das aplicações. Entretanto, pouco tem sido feito para orquestrar quais e com qual frequência os itens de telemetria devem ser coletados. Os artigos apresentados abaixo discutem os avanços mais recentes relacionados com a utilização de telemetria in-band.

[Jeyakumar et al. 2014] propõe que instruções simples sejam encapsuladas em pacotes e possam ser executadas em dispositivo programáveis para identificar problemas como micro-burst e congestionamento de rede. Para isso, considera-se que cada dispositivo seja responsável por implementar funções bem definidas: (a) switches programáveis encaminham e executam programas em pacotes Ethernet modificados - chamados de Tiny Packet Programs (TPPs), enquanto (b) hosts computam informações sobre o estado da rede, interagindo com o plano de controle quando necessário. Utilizando um dispositivo NetFPGA, observa-se um overhead baixo na inclusão das instruções nos TPPs, porém a variedade de aplicações possíveis nos TPPs ainda é restrita ao (i) conjunto de instruções existentes e (ii) e às próprias requisições - já que estas devem ser inicializadas pelos hosts.

EverFlow [Zhu et al. 2015] e PathDump [Tammana et al. 2016] coletam informações em infraestruturas de rede de Data Centers (DCN - Datacenter Network) para auxiliar no debugging e gerenciamento da infraestrutura, identificando a origem de falhas que podem causar problemas de roteamento, balanceamento de carga e potenciais loops. Ambos os trabalhos utilizam dispositivos de encaminhamento não programáveis para rastrear o caminho dos pacotes através da insfraestrutura de rede. EverFlow [Zhu et al. 2015] prioriza a coleta de dados de telemetria a partir dos dispositivos de encaminhamento, enquanto que PathDump [Tammana et al. 2016] visa distribuir essa função entre hosts e os próprios dispositivos de encaminhamento. Em suma, ambos são capazes de realizar diagnósticos sobre uma quantidade similar de problemas.

Por sua vez, [Jianzhe Liang 2018] objetiva a solução de problemas (isto é, trou- 
bleshooting) em infraestruturas genéricas de rede. Para isso, verifica-se as SLAs das funções de rede existentes através da coleta de metadados utilizando telemetria in-band ou através de postcards. Considera que existem três categorias de nós de telemetria: (a) classificador, (b) encaminhador e (c) coletor. Os dispositivos classificadores inserem headers de telemetria em determinados pacotes que, por sua vez, recebem dos encaminhadores os metadados do estado da rede que são enviadas para os coletores - onde, por fim, os metadados são extraídos e utilizados. Sonata [Gupta et al. 2018] e NetVision [Liu et al. 2018] também consideram que cada nó da infraestrutura de rede desempenha uma determinada função no processo de coleta dos metadados da rede. Sonata fornece uma interface declarativa para expressar requisições de telemetria em alto nível, as quais são compiladas e executadas nos dispositivos da infraestrutura de rede. Por sua vez, NetVision fornece uma interface que é responsável por abstrair as requisições de telemetria e gerar pacotes probes que são encaminhados através de caminhos pré-computados na infraestrutura para coletarem os dados de telemetria.

Apesar das abordagens existentes realizarem esforços para diagnosticar diferentes problemas na rede, pouco ainda foi feito para orquestrar a forma com que itens de telemetria podem ser coletados. Como mencionado anteriormente, o trabalho de [Marques et al. 2019] é o primeiro esforço nesta direção. [Marques et al. 2019] propõe duas heurísticas, nomeadas de Concentra e Distribui, para orquestrar como dados de telemetria in-band são coletados. Na heurística Concentra, os itens de telemetria disponíveis são encapsulados no menor número de fluxos possíveis, enquanto que na estratégia Distribui, os itens de telemetria são distribuídos entre diferentes fluxos de maneira uniforme.

\section{Definição do Problema}

Nesta seção, inicialmente apresenta-se uma descrição geral para o problema de orquestração da telemetria in-band e, em seguida, uma definição formal. O conceito de telemetria in-band discutido neste artigo, consiste na inserção de informações de monitoramento (por exemplo, número de pacotes, atraso no encaminhamento) dentro dos fluxos que trafegam pela rede. Essas informações podem ser inseridas durante o encaminhamento dos pacotes aproveitando espaços não utilizados nos cabeçalhos (por exemplo, no campo type of service do protocolo IPv4), sendo extraídas antes da entrega dos dados. A inserção de informações dentro dos pacotes resulta em um maior tempo de processamento desses pacotes nos dispositivos de encaminhamento e, portanto, essa inserção deve ser orquestrada de maneira eficiente.

O modelo de otimização proposto considera uma infraestrutura de rede física representada por um grafo $G=(D, L)$, onde $D$ representa os dispositivos programáveis de encaminhamento $D=\left\{d_{1}, d_{2}, \ldots, d_{|D|}\right\}$ e $L$ representa os enlaces entre pares de dispositivos $\left(d_{1}, d_{2}\right) \in(D \times D)$. Além disso, considera-se a existência de um conjunto de fluxos ativos $F$ e um conjunto de itens de telemetria $V$. Todo dispositivo de encaminhamento $d \in D$ é capaz de embutir um subconjunto de itens $V_{d} \in V$ nos pacotes de um fluxo $f \in F$. O número de itens $V_{d} \in V$ embutidos nos pacotes de um fluxo $f \in F$ é limitado pelo tamanho de cada item, que é definido pela função $S: V \rightarrow \mathbb{N}^{+}$, e pela capacidade do fluxo, definida pela constante $K_{f}$. Cada fluxo $f \in F$ consiste em um conjunto de pacotes encaminhados entre dois dispositivos $d \in D$ finais (origem e destino), e roteados através da infraestrutura $G$ utilizando um caminho simples. A função $\mathcal{P} t: F \rightarrow\left\{D_{1} \times \ldots \times D_{|D|}\right\}$ denota o caminho utilizado pelo fluxo $f \in F$. Durante o encaminhamento dos pacotes de um fluxo $f \in F$, os pacotes são encapsulados utilizando protocolos de roteamento (por exemplo, NSH [P. Quinn 2018], IPv4). Esses protocolos, em geral, não utilizam totalmente o espaço de seus cabeçalhos (por exemplo, type of service do protocolo IP), possibilitando que os itens de telemetria sejam encapsulados nesses espaços. Portanto, $K_{f}$ é o espaço disponível para inserção de itens de telemetria em um fluxo $f \in F$.

O problema de otimização consiste em uma infraestrutura de rede $G$, um conjunto de fluxos $F$, um conjunto de itens $V$ e um conjunto de constante $K_{f}$. A solução deve 
maximizar o número de itens de telemetria coletados (descrito pela Equação (1)). A saída do modelo de otimização consiste em $Y$, sendo $Y=\left\{y_{d, v, f}, \forall d \in D, v \in V, f \in F\right\}$ onde as variáveis $y \in Y$ representam se um dispositivo de encaminhamento $d$ encapsula o item de telemetria $v$ em pacotes do fluxo $f$. O modelo de otimização é descrito a seguir.

$$
\text { Maximizar } \quad \sum_{d \in D} \sum_{v \in V} \sum_{f \in F} y_{d, v, f}
$$

\section{Sujeito a:}

$$
\begin{array}{lr}
\sum_{d \in \mathcal{P} t(f)} \sum_{v \in V_{d}} y_{d, v, f} \cdot S(v) \leq K_{f} & \forall f \in F \\
\sum_{f \in F} y_{d, v, f} \leq 1 & \forall d \in D, v \in V_{d}, \\
y_{d, v, f} \in\{0,1\} & \forall d \in D, v \in V, f \in F
\end{array}
$$

O conjunto de restrições (2) garante que um fluxo de rede $f \in F$ não exceda sua capacidade ao coletar itens de telemetria. Por sua vez, o conjunto de restrições (3) garante que um único item de telemetria seja coletado no máximo uma vez por um determinado fluxo $f \in F$. Por fim, o conjunto de restrições (4) define o domínio das variáveis do modelo.

\section{Heurísticas Propostas}

A seguir, descreve-se as duas heurísticas propostas neste trabalho para a coleta de dados de telemetria in-band. Ambas as heurísticas objetivam maximizar os itens de telemetria coletados ao longo do tempo seguindo estratégias locais diferentes. A primeira, baseia-se no conceito Least Recently Colected (LRC), enquanto que a segunda segue a estratégia Round-Robin (RR).

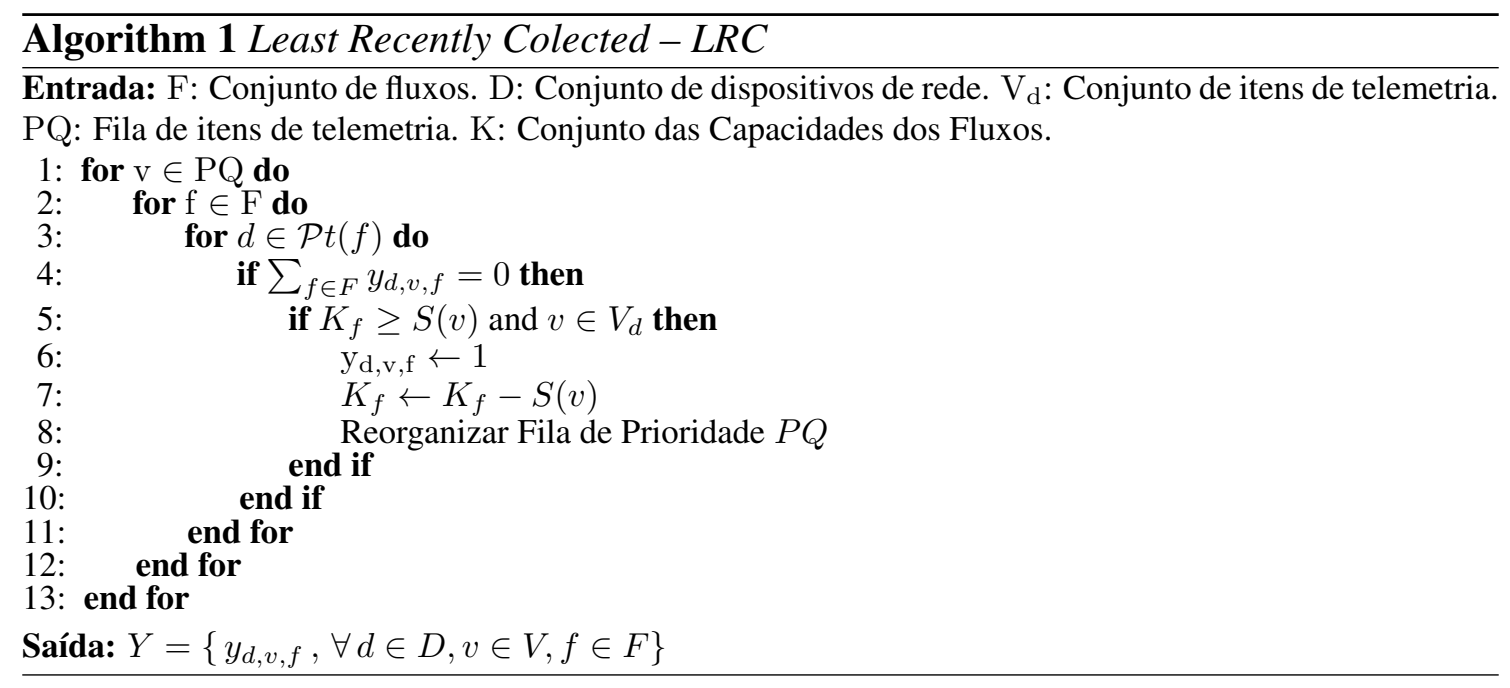

A heurística LRC prioriza a coleta de itens de telemetria de forma a minimizar o tempo médio de coleta ao longo do tempo. Os itens que foram coletados menos recentemente em relação com a unidade de tempo atual tem preferência para serem coletados na próxima unidade de tempo. O Algoritmo 1 ilustra o pseudocódigo da estratégia proposta. 
O algoritmo recebe como entrada o conjunto de fluxos ativos $F$, o conjunto de dispositivos de encaminhamento $D$ com seus respectivos itens de telemetria $V_{d}$, uma fila de prioridade de itens (ordenada do item menos recentemente coletado ao mais recente) e o conjunto $K_{f}$. A saída do algoritmo é representada pelo conjunto de variáveis $Y$. Na linha 1 , percorre-se a fila de itens priorizando a coleta dos itens de telemetria que foram menos recentemente coletados. Nas linhas 2 e 3, percorre-se o conjunto de dispositivos de encaminhamento pelos quais o fluxo $f \in F$ é roteado. Na linha 4 , verifica-se se o item $v$ foi coletado por algum fluxo ativo, enquanto que na linha 5 , verifica-se se a disponibilidade do item $v$ no dispositivo $d$ e se o fluxo $f$ tem capacidade $K_{f}$ para coletar o mesmo. Caso o item seja coletado, decrementa-se a capacidade disponível no fluxo e reorganiza-se a fila de prioridade - posicionando o item coletado ao fim da fila.

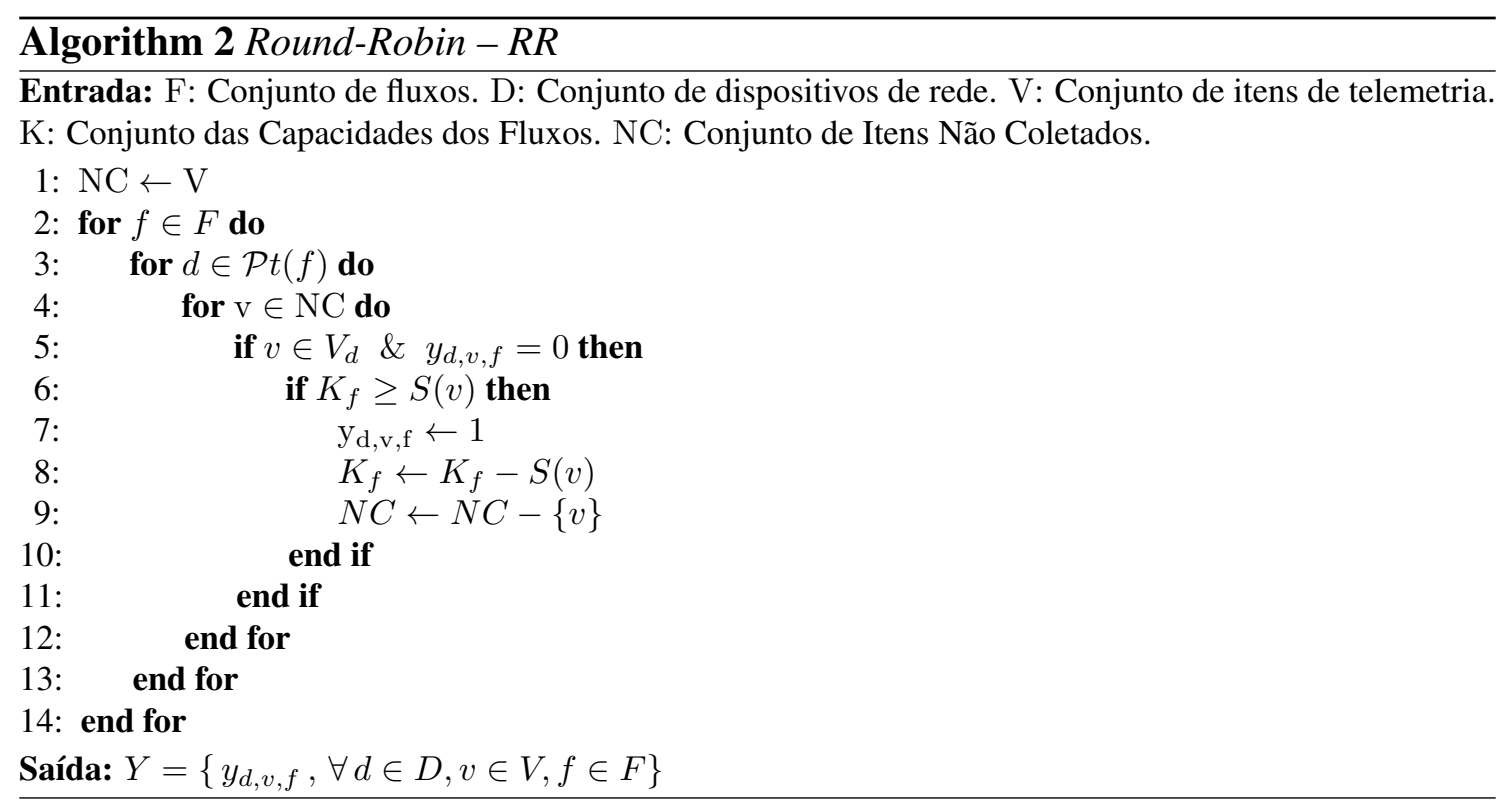

O Algoritmo 2 ilustra a segunda estratégia proposta, a qual é baseada em RoundRobin. A heurística busca equalizar a coleta de itens de telemetria ao longo do tempo, distribuindo igualmente a carga entre os fluxos. Na linha 1, o conjunto $N C$ representa os itens de telemetria não coletados. As linhas 2 e 3 percorrem os dispositivos de encaminhamento pelos quais os fluxos ativos são roteados. As linhas 4, 5 e 6 , verificam se o item ainda não foi coletado e se há capacidade no fluxo, coletando-o ou não. Após coletado, o mesmo é retirado do conjunto de itens não coletados $(N C)$.

\section{Análise das Heurísticas Propostas}

Nesta seção, descreve-se a carga de trabalho e os resultados da avaliação das heurísticas propostas. Os experimentos foram realizados em uma máquina com um processador Intel i7 7700 com 16 GB de memória RAM, usando o sistema operacional Ubuntu 14.04 LTS.

\subsection{Carga de Trabalho}

Para fins de análise, foi desenvolvido um simulador para realizar a coleta de itens de telemetria in-band, executando por um período de 50 unidades de tempo. Em cada unidade de tempo, os algoritmos são executados para determinar quais fluxos coletam quais itens de telemetria. Considera-se uma topologia de rede Hub and Spoke [Luizelli et al. 2016] com 50 dispositivos de encaminhamento e, em média, 200 enlaces. Cada dispositivo de encaminhamento possui 8 itens de telemetria possíveis de serem coletados com tamanhos variando de 1 a 30 Bytes. Considera-se a existência de um conjunto de fluxos de rede ativos $F$ sobre a infraestrutura. Varia-se a quantidade de fluxos ativos de 50 a 600 . Cada 


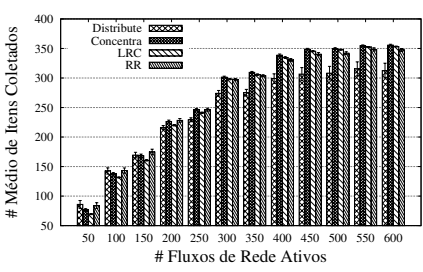

(a) Quantidade de itens coletados.

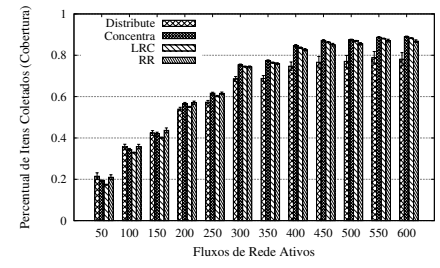

(b) Cobertura de rede.

Figura 1. Quantidade de itens coletados e cobertura da rede.

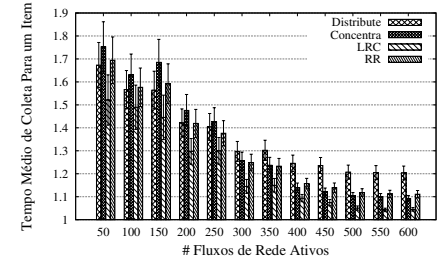

(a) Tempo médio de coleta de um item.

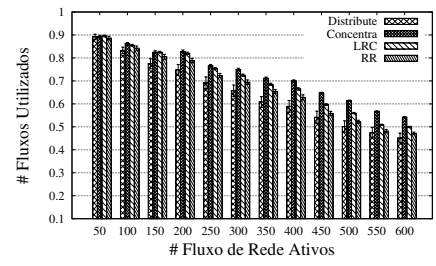

(b) Quantidade de fluxos utilizados.

Figura 2. Tempo médio de coleta de um item e quantidade de fluxos utilizados.

fluxo $f \in F$ interconecta dois dispositivos de encaminhamento (escolhidos de maneira aleatória) e são roteados utilizando o caminho mínimo. Cada fluxo $f \in F$ tem sua capacidade $K_{f}$ uniformemente distribuída entre 1 e 30 Bytes. Compare-se as heurísticas propostas (LRC e RR) com as duas heurísticas de [Marques et al. 2019]: Concentra e Distribui. Foram executadas 30 repetições de cada experimento.

\subsection{Resultados}

Primeiramente, avalia-se a quantidade de itens de telemetria coletados e a cobertura média em uma unidade de tempo. A Figura 1(a) ilustra a quantidade média de itens coletados em cada cenário avaliado. Observa-se que o número de fluxos ativos impacta diretamente na quantidade de itens de telemetria coletados. No geral, quanto maior o número de fluxos ativos, maior será o número de itens coletados. É possível observar que este comportamento se mantem até 400 fluxos de rede ativos. Após, o aumento de fluxos impacta superficialmente no volume de dados coletados. Este comportamento está diretamente relacionado com as estratégias algorítmicas adotadas, a topologia de rede e o roteamento dos fluxos. No geral, a maior parte dos fluxos ativos são encaminhados através de dispositivos de encaminhamento com maior interconexão. Dessa forma, uma parte dos itens de telemetria podem ficar cobertas por um conjunto reduzido de fluxos ativos. Ainda, como as estratégias avaliadas são gulosas, as decisões tomadas pelos algoritmos podem inviabilizar a coleta de alguns itens em uma determinada unidade de tempo. Por sua vez, a Figura 1(b) ilustra a cobertura dos itens coletados. Esta métrica mostra o percentual de itens coletados por unidade de tempo para cada uma das estratégias avaliadas e está diretamente relacionada com os dados ilustrados na Figura 1(a). É possível observar o número de itens coletados e, consequentemente, a cobertura de itens é similar para os métodos LRC, RR e Concentra (em torno de 87\%). Entretanto, o método Distribui apresenta uma cobertura inferior a $80 \%$ dos itens.

A Figura 2(a) ilustra o tempo médio de coleta de um item, em unidades de tempo para cada um dos cenários avaliados. Ressalta-se que, com tempos médios menores, a frequência de coleta dos itens de telemetria aumenta. Nos gráficos, valores próximos ao valor 1 representam o cenário ideal. Observa-se que o aumento do número de fluxos ativos reduz significativamente o tempo médio de coleta. Para 50 fluxos de rede ativos, 


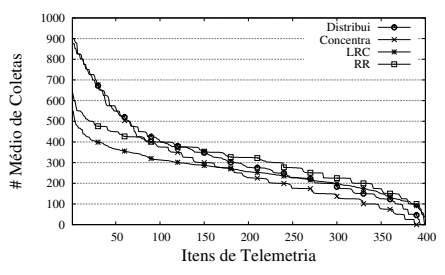

(a) 50 fluxos ativos.

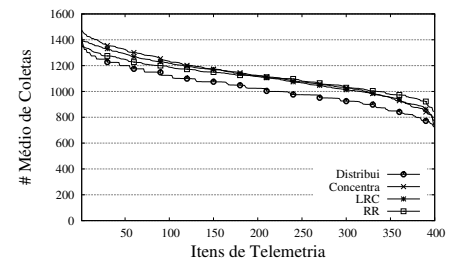

(b) 300 fluxos ativos.

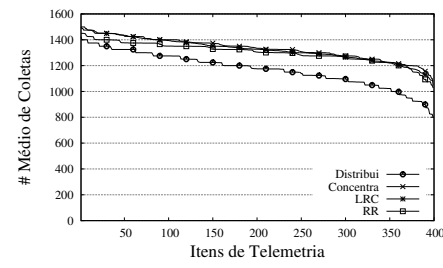

(c) 600 fluxos ativos.

Figura 3. Quantidade de coletas para cada item variando o número de fluxos.

o valor médio do intervalo entre as coletas dos itens de telemetria é de 1.6 unidades de tempo (isto é, $60 \%$ maior que o ideal). À medida que o número de fluxos aumenta (por exemplo, para 500 fluxos ativos), o valor médio é reduzido para próximo de 1 unidade de tempo. Com o aumento do número de fluxos ativos, aumenta-se a probabilidade de que estes fluxos coletem dados de telemetria. Isto se dá já que o espaço de soluções factíveis explorado pelas heurísticas aumenta consideravelmente - facilitando a coleta de itens de telemetria, mesmo em dispositivos menos interconectados. Observa-se que a heurística LRC apresenta o menor tempo de coleta para os itens. Para 50 fluxos de redes, o LRC reduz em até $15 \%$ o valor médio do tempo entre coletas em relação à heurística Concentra.

A seguir, avalia-se a quantidade de fluxos de rede utilizados. A Figura 2(b) ilustra a quantidade de fluxos de rede disponíveis versus a quantidade de fluxos de rede utilizados para a coleta de dados de telemetria. Como é possível observar, à medida que o número de fluxos de rede aumenta, o percentual de fluxos de rede em uso para a coleta de dados diminui linearmente. Esse comportamento está diretamente relacionado com a topologia e com a estratégia de roteamento. Como mencionado anteriormente, o roteamento adotado pelos fluxos segue o menor caminho e, portanto, muitos fluxos tendem a serem roteados por um subconjunto restrito de dispositivos e enlaces. Apesar do aumento do espaço de soluções factíveis gerado pelo aumento de fluxos, o espaço de soluções factíveis não aumenta de maneira proporcional em todas as regiões da infraestrutura. Dessa forma, fluxos de rede que são roteados por dispositivos de encaminhamento altamente interconectados tendem a ficar ociosos com o aumento de fluxos da rede (já que a oferta de fluxos nesses pontos aumenta, mas a demanda continua a mesma).

Por fim, avalia-se o número médio absoluto de vezes que um determinado item de telemetria é coletado ao longo das 50 unidades de tempo. A Figura 3 ilustra, para cada item de telemetria (isto é, de 1 a 400), a quantidade de vezes média que foi coletado. Os itens de telemetria foram ordenados em relação ao número de vezes em que foram coletados. As Figuras 3(a), 3(b) e 3(c) ilustram, respectivamente, os cenários avaliados para 50,

300 e 600 fluxos de rede ativos. É possível observar que alguns itens de telemetria são coletados com maior intensidade do que outros. Para cenários onde o nível de atividade da rede é baixo (por exemplo, 50 fluxos ativos), a discrepância absoluta de coletas entre itens é maior, podendo chegar até 900x para os métodos Distribui e Concentra. Os métodos propostos LRC e RR apresentam menor discrepância absoluta no número de coletas de até $50 \%$ em relação aos métodos Distribui e Concentra. Ao aumentar o nível de atividade da rede (casos de 300 e 600 fluxos ativos), a diferença absoluta entre o número de coletas reduz. Entretanto, é possível observar que alguns itens de telemetria são coletados até $1.75 x$ e $1.66 x$ mais do que outros - respectivamente para 300 e 600 fluxos.

\section{Conclusão}

Neste artigo, foram propostas duas heurísticas online - LRU e RR - para o problema de coleta de itens de telemetria in-band. A partir da análise do comportamento das heurísticas, observou-se que conforme o número de fluxos ativos aumenta na infraestrutura, aumenta-se a probabilidade de que os itens sejam coletados em menor tempo. 
Além disso, a utilização dos fluxos ativos é limitada pelas características topológicas da infraestrutura e pelas estratégias de roteamento adotado pelos fluxos. Espera-se avaliar as heurísticas em outras topologias de rede e desenvolver novos mecanismos de roteamento que sejam cientes da coleta de itens de telemetria e das limitações topológicas.

\section{Referências}

Gulenko, A., Wallschläger, M., and Kao, O. (2018). A practical implementation of inband network telemetry in open vswitch. In 2018 IEEE 7th International Conference on Cloud Networking (CloudNet), pages 1-4. IEEE.

Gupta, A., Harrison, R., Canini, M., Feamster, N., Rexford, J., and Willinger, W. (2018). Sonata: Query-driven streaming network telemetry. In Proceedings of the 2018 ACM Conference on SIGCOMM, SIGCOMM '18, New York, NY, USA. ACM.

Jeyakumar, V., Alizadeh, M., Geng, Y., Kim, C., and Mazières, D. (2014). Millions of little minions: Using packets for low latency network programming and visibility. In Proceedings of the 2014 ACM Conference on SIGCOMM, SIGCOMM '14, pages 3-14, New York, NY, USA. ACM.

Jianzhe Liang, Jun Bi, Y. Z. C. Z. (2018). In-band network function telemetry. In Proceedings of the Poster 2018 ACM Conference on SIGCOMM, SIGCOMM '18, pages $1-4$, New York, NY, USA. ACM.

Liu, Z., Bi, J., Zhou, Y., Wang, Y., and Lin, Y. (2018). Netvision: Towards network telemetry as a service. In 2018 IEEE 26th International Conference on Network Protocols (ICNP), pages 247-248. IEEE.

Liu, Z., Manousis, A., Vorsanger, G., Sekar, V., and Braverman, V. (2016). One sketch to rule them all: Rethinking network flow monitoring with univmon. In Proceedings of the 2016 ACM Conference on SIGCOMM, SIGCOMM '16, pages 101-114, New York, NY, USA. ACM.

Luizelli, M. C., Bays, L. R., Buriol, L. S., Barcellos, M. P., and Gaspary, L. P. (2016). How physical network topologies affect virtual network embedding quality: A characterization study based on $\{$ ISP $\}$ and datacenter networks. Journal of Network and Computer Applications, 70:1 - 16.

Marques, J. A., Luizelli, M. C., Tavares da Costa Filho, R. I., and Gaspary, L. P. (2019). An optimization-based approach for efficient network monitoring using inband network telemetry. Journal of Internet Services and Applications, 10(1):12.

P. Quinn, Ed.; U. Elzur, E. E. (2018). Network service header (nsh). Internet Draft.

Putina, A., Rossi, D., Bifet, A., Barth, S., Pletcher, D., Precup, C., and Nivaggioli, P. (2018). Telemetry-based stream-learning of bgp anomalies. In Proceedings of the 2018 Workshop on Big Data Analytics and Machine Learning for Data Communication Networks, Big-DAMA '18, pages 15-20, New York, NY, USA. ACM.

Q. Wu, J. Strassner, A. F. and Zhang., L. (2016). Network telemetry and big data analysis.

Tammana, P., Agarwal, R., and Lee, M. (2016). Simplifying datacenter network debugging with pathdump. In 12th USENIX Symposium on Operating Systems Design and Implementation (OSDI 16), pages 233-248, Savannah, GA.

Tammana, P., Agarwal, R., and Lee, M. (2018). Distributed network monitoring and debugging with switchpointer. In 15th USENIX Symposium on Networked Systems Design and Implementation (NSDI 18), pages 453-456, Renton, WA.

Zhu, Y., Kang, N., Cao, J., Greenberg, A., Lu, G., Mahajan, R., Maltz, D., Yuan, L., Zhang, M., Zhao, B. Y., and Zheng, H. (2015). Packet-level telemetry in large datacenter networks. In Proceedings of the 2015 ACM Conference on SIGCOMM, SIGCOMM '15, pages 479-491, New York, NY, USA. ACM. 